\title{
Microbiota Plays a Key Role in Non-Steroidal Anti-Inflammatory Drug-Induced Small Intestinal Damage
}

\author{
Koji Otani $^{\mathrm{a}}$ Tetsuya Tanigawa ${ }^{\mathrm{a}, \mathrm{b}}$ Toshio Watanabe ${ }^{\mathrm{a}, \mathrm{b}}$ Sunao Shimada ${ }^{\mathrm{a}}$ \\ Yuji Nadatani $^{a}$ Yasuaki Nagami ${ }^{\mathrm{a}}$ Fumio Tanaka ${ }^{\mathrm{a}}$ Noriko Kamata ${ }^{\mathrm{a}}$ \\ Hirokazu Yamagami ${ }^{a}$ Masatsugu Shiba ${ }^{a}$ Kazunari Tominaga ${ }^{a, b}$ \\ Yasuhiro Fujiwara $^{a, b}$ Tetsuo Arakawa ${ }^{a}$
}

${ }^{a}$ Department of Gastroenterology, and b Samurai International GI Research Centre, Osaka City University Graduate

School of Medicine, Osaka, Japan

\section{Key Words}

Toll-like receptor - High mobility group box 1 .

Nucleotide-binding oligomerization domain-like receptor

family Pyrin domain-containing $3 \cdot$ Inflammasome .

Probiotics · Rebamipide

\section{Abstract \\ Background: Non-steroidal anti-inflammatory drugs (NSAIDs) damage the small intestine by causing multiple erosions and ulcers. However, to date, no established thera- pies and prophylactic agents are available to treat such dam- ages. We reviewed the role of intestinal microbiota in NSAID- induced intestinal damage and identified potential thera- peutic candidates. Summary: The composition of the intestinal microbiota is an important factor in the patho- physiology of NSAID-induced small intestinal damage. Once mucosal barrier function is disrupted due to NSAID-induced prostaglandin deficiency and mitochondrial malfunction, li- popolysaccharide from luminal gram-negative bacteria and high mobility group box 1 from the injured epithelial cells activate toll-like receptor 4-signaling pathway and nucleo- tide-binding oligomerization domain-like receptor family,}

pyrin domain-containing 3 inflammasome; this leads to the release of proinflammatory cytokines such as tumor necrosis factor- $\alpha$ and interleukin- $1 \beta$. Proton pump inhibitors (PPIs) are often used for the prevention of NSAID-induced injuries to the upper gastrointestinal tract. However, several studies indicate that PPIs may induce dysbiosis, which may exacerbate the NSAID-induced small intestinal damage. Our recent research suggests that probiotics and rebamipide could be used to prevent NSAID-induced small intestinal damage by regulating the intestinal microbiota. Key Messages: Intestinal microbiota plays a key role in NSAID-induced small intestinal damage, and modulating the composition of the intestinal microbiota could be a new therapeutic strategy for treating this damage.

(c) 2016 S. Karger AG, Basel

\section{Introduction}

Currently, non-steroidal anti-inflammatory drugs (NSAIDs) constitute one of the most frequently prescribed class of drugs. It is known that NSAIDs damage the mucosa of the upper gastrointestinal (GI) tract; more-

\section{KARGER}

E-Mail karger@karger.com www.karger.com/dig
C 2016 S. Karger AG, Basel

0012-2823/16/0951-0022\$39.50/0 

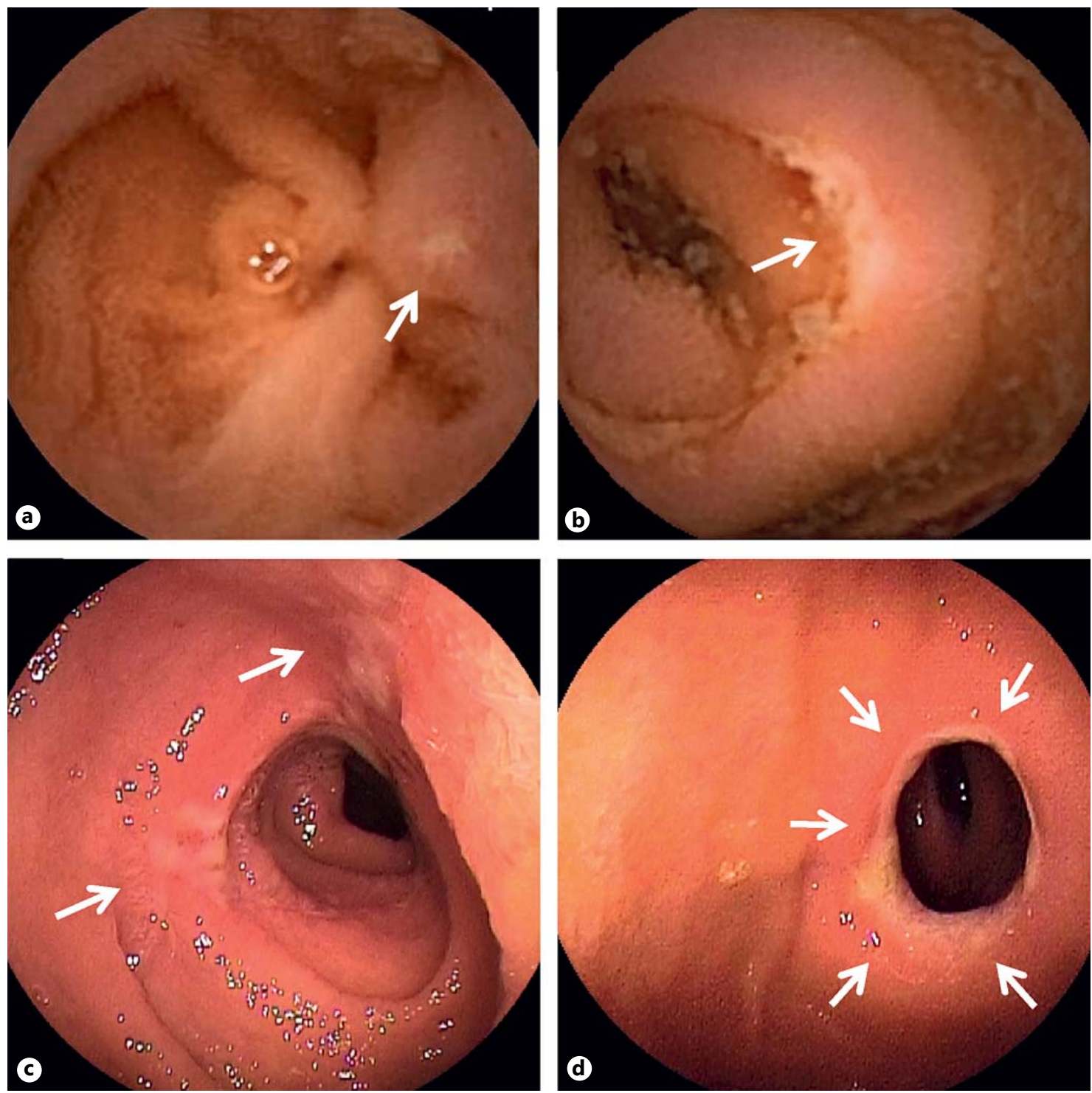

Fig. 1. Typical images of NSAID-induced small intestinal damage detected using capsule endoscopy and balloon-assisted endoscopy. a Erosion detected by video capsule endoscopy (arrow). b Ulcer detected by video capsule endoscopy (arrow). c Irregular ulcers detected by balloon-assisted enteroscopy (arrows). d Circular ulcer detected by balloon-assisted enteroscopy (arrows). over, several studies using video capsule endoscopy and balloon-assisted enteroscopy show that NSAIDs damage the small intestine [1-3]. NSAIDs, including low-dose aspirin (LDA), induce multiple mucosal erosions and ulcers across the small intestine, which cause small intestinal bleeding and obstruction (fig. 1).

NSAIDs cause damage to the upper GI tract through the inhibition of cyclooxygenase and subsequent deficiency of prostaglandin (PG), which plays an important role in the mucosal defense system. Similarly, NSAIDinduced small intestinal damage is also considered to be caused due to PG deficiency. However, the pathophysiology of small intestinal damage is different from that of upper GI damage, as the small intestine is independent of gastric acid. Although acid suppressants such as proton pump inhibitors (PPIs) and histamine $\mathrm{H}_{2}$-receptor antagonists prevent NSAID-induced damages to the upper GI tract, these agents are not effective against small intestinal damage, as hydrochloric acid is not produced in the small intestine and gastric acid is neutralized by bicarbonate in the duodenum. Intestinal microbiota is considered to play a key role in the 
pathophysiology of NSAID-induced small intestinal damage.

We reviewed findings from recent studies and discussed the involvement of the intestinal microbiota in the pathophysiology of NSAID-induced intestinal damage; we also put forward a strategy for the prevention and treatment of such damage.

\section{Microbial Composition in the Small Intestine}

Gu et al. [4] determined the composition and diversity of microorganisms in the GI tract of mice by using highthroughput pyrosequencing analysis. Their results showed that the small intestine had a high proportion (approximately 25\%) of microorganisms belonging to the family Lactobacillaceae, whereas the colon and feces had a high proportion of microorganisms belonging to the families Bacteroidaceae, Prevotellaceae, Rikenellaceae, Lachnospiraceae, and Ruminococcaceae. The environment in the GI tract affected the dominance of bacteria in the different regions of the GI tract. The levels of oxygen in the stomach and small intestine are higher than those in the colon. Thus, high levels of facultative anaerobic bacteria, including Lactobacillaceae, were present in the small intestine and strictly anaerobic bacteria were present in the colon.

Compared to the data on intestinal microbiota in experimental animal, the data in humans is limited because of the clinical difficulty in accessing this region. Zoetendal et al. [5] reported that bacteria from the phylum Bacteroidetes, Clostridium subcluster XIVa, and the phylum Proteobacteria were dominant in the samples from the ileal fluid, and those from the class Bacilli, Clostridium subclusters IX and XIVa, and the class Gamma-proteobacteria were dominant in the samples from the jejunal fluid collected by an intraluminal naso-ileal catheter from healthy volunteers using the GI tract-specific phylogenetic microarray analysis.

\section{Involvement of Bacteria in NSAID-Induced Small Intestinal Damage}

The involvement of bacteria in NSAID-induced small intestinal damage was observed about 40 years ago. Robert and Asano [6] reported that germ-free rats were resistant to indomethacin-induced small intestinal damage, while germ-free rats exposed to Escherichia coli developed severe damages to the small intestine. Uejima et al. [7] showed that germ-free rats and gnotobiotic rats mono-associated with Bifidobacterium or Lactobacillus had no intestinal ulcers after administration of NSAIDs, whereas NSAIDs induced ileal ulcers in gnotobiotic rats mono-associated with Eubacterium limosum or E. coli. Moreover, Konaka et al. [8] showed that ampicillin, an antibacterial agent against gram-positive and gram-negative bacteria, inhibits indomethacin-induced small intestinal damages and decreases the number of enterobacteria in rats. These experimental results clearly show that the composition of the intestinal microbiota is an important factor in NSAID-induced enteropathy.

Takeuchi and Satoh [9] showed that enterobacterial invasion is the most important pathogenic event for the development of NSAID-induced enteropathy though multiple factors such as intestinal hypermotility, decreased mucus secretion, and upregulation of inducible nitric oxide synthase/nitric oxide.

\section{Role of Toll-Like Receptor 4 in NSAID-Induced Small Intestinal Damage}

The mucosa of the small intestine is exposed to various types of danger signals, including pathogen-associated molecular patterns (PAMPs) and damage-associated molecular patterns (DAMPs), and the innate immune system is activated when these danger signals are recognized by pattern-recognition receptors such as toll-like receptors (TLRs) and nucleotide-binding oligomerization domain-like receptors (NLRs).

The TLRs specifically recognize the components of pathogenic microorganisms, and TLR4 recognizes lipopolysaccharide (LPS), which is a membrane component found in gram-negative bacteria and acts as a PAMP. Previously, we showed that the NSAID-triggered inflammatory response in the small intestine was through the TLR4/ myeloid differentiation primary-response 88 (MyD88)signaling pathway [10]. Indomethacin-induced small intestinal damage was less in TLR4-mutant mice with the suppression of tumor necrosis factor- $\alpha$ (TNF- $\alpha$ ) and monocyte chemotactic protein-1. Further, the damage to

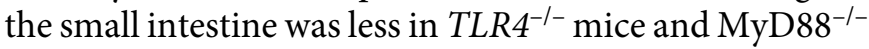
mice. In addition, we reported that high mobility group box 1 (HMGB1), a nuclear protein that can bind to DNA and act as a co-factor for gene transcription and as a DAMP, is released from injured epithelial cells and binds to TLR4, which results in the activation of nuclear factor$\kappa \mathrm{B}(\mathrm{NF}-\kappa \mathrm{B})$ through the MyD88-dependent pathway in NSAID-induced small intestinal damage [11]. 
Fig. 2. The mechanism underlying the pathophysiology of NSAID-induced intestinal damage.

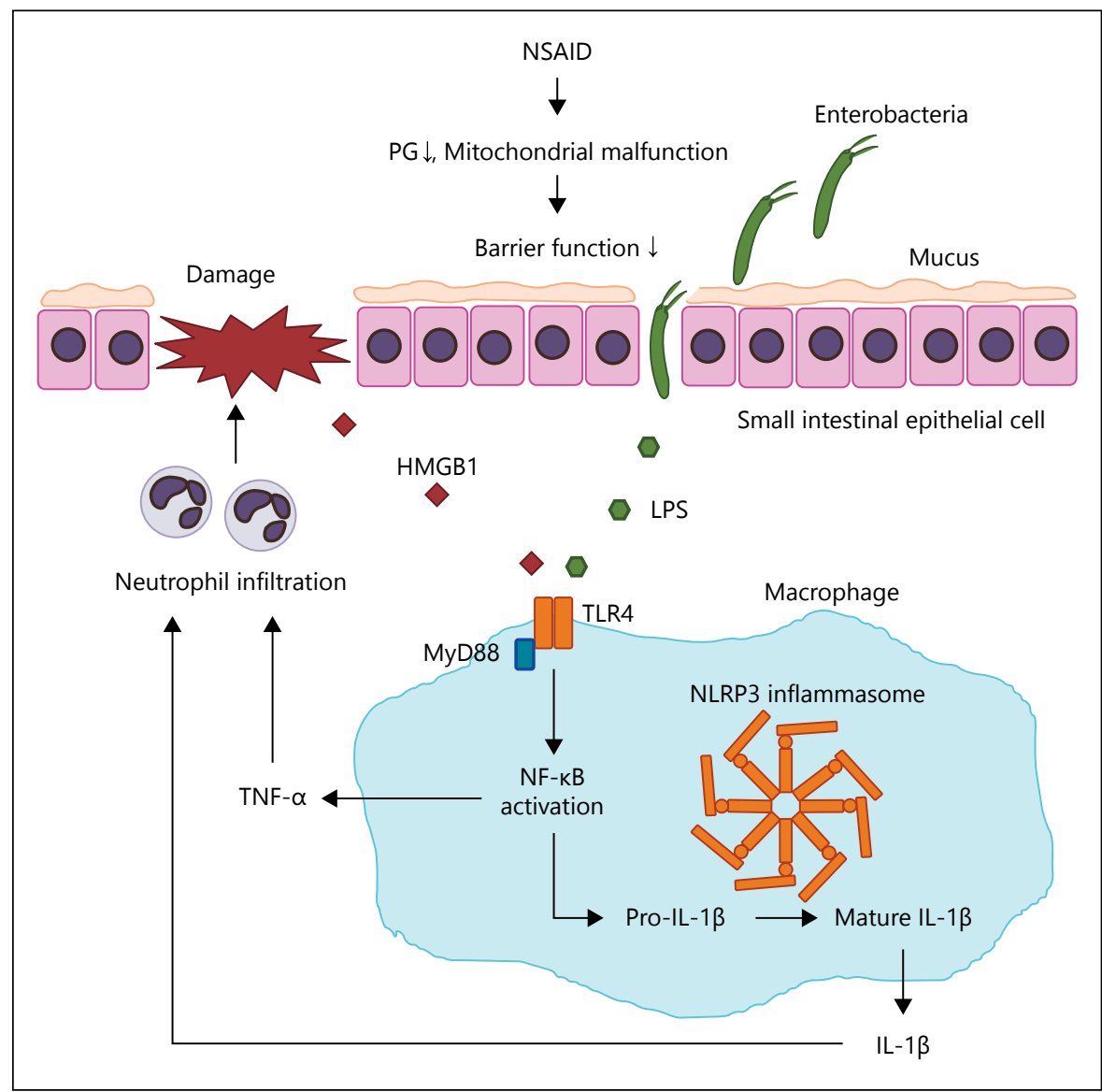

NLRs are present inside the cell membrane and their activation by danger signals causes the formation of an inflammasome. Activation of the inflammasome results in the cleavage of pro-caspase- 1 to the cleaved form of caspase-1, which promotes the processing of prointerleukin-1 $\beta$ (IL-1 $\beta$ ) into mature IL- $1 \beta$. The NLR family, pyrin domain-containing 3 (NLRP3), also known as NALP3 or cryopyrin, is one of the most investigated molecules among the inflammasomes, and we previously reported that mature IL- $1 \beta$ induced by activated NLRP3 inflammasome acted as an important proinflammatory cytokine as well as TNF- $\alpha$ in the pathophysiology of NSAID-induced small intestinal damage [12].

On the basis of these results, we propose the mechanism underlying the pathophysiology of NSAID-induced intestinal damage. Once mucosal barrier function is disrupted due to NSAID-induced PG deficiency and mitochondrial malfunction, LPS from luminal gramnegative bacteria and HMGB1 from the injured epithelial cells bind to TLR4 on macrophages; this results in the activation of NF- $\kappa \mathrm{B}$ through MyD88-dependent path- way and the NLRP3 inflammasome [13]. This leads to the release of proinflammatory cytokines such as TNF- $\alpha$ and IL-1 $\beta$ and chemokines, which induce neutrophil infiltration into the mucosa and submucosa of the small intestine, thus causing damage to the small intestine through the production and release of cytotoxic chemicals by neutrophils such as reactive oxygen species and elastase (fig. 2).

\section{Significance of Dysbiosis in NSAID-Induced Small Intestinal Damage}

The term 'dysbiosis' was first used by Bai [14] in 1985. Dysbiosis is an abnormality present in the composition of the microbial community in which the population of bacteria beneficial for the host health decreases and the population of pathogenic bacteria typically present in small numbers increases. In the GI tract, dysbiosis has mostly been studied with regard to the inflammatory bowel disease $[15,16]$. Dysbiosis is associated with various types of 
diseases such as diabetes mellitus [17], obesity [18], and non-alcoholic fatty liver disease [19].

Small intestinal bacterial overgrowth (SIBO) is a type of dysbiosis that indicates the presence of excessive bacteria in the small intestine and is defined as the presence of bacteria $\geq 10^{5}$ colony forming units $/ \mathrm{ml}$ in the jejunum with symptoms or malabsorption [20]. We assessed the association between the severity of NSAID-induced small intestinal damage and bacterial overgrowth measured by lactulose hydrogen breath test (LHBT) in patients with rheumatoid arthritis and osteoarthritis using NSAIDs for more than 3 months [21]. Our results showed that LHBT-positive patients were significantly associated with an increased odds ratio for severe NSAID-induced small intestinal damage. These results suggest that SIBO is a risk factor for NSAID-induced severe small intestinal damage.

Wallace et al. [22] reported that PPIs induce dysbiosis and exacerbate the NSAID-induced small intestinal damage in rats. Administration of PPIs (omeprazole and lansoprazole) exacerbated NSAID-induced small intestinal damages, accompanied by reduction in the proportion of Actinobacteria and Bifidobacteria spp in rats. Recolonization with Bifidobacteria-enriched commensal bacteria prevented the NSAID and PPI-induced small intestinal damages and bleeding, whereas colonization with bacteria from PPI-treated rats developed the NSAID-induced small intestinal damage in germ-free mice. These results indicated that PPIs exacerbate the NSAID-induced small intestinal damage by changing the enteric microbial populations.

Consistent with the results of this experimental study, the results of our previous clinical study showed that PPIs were the risk factors of NSAID-induced severe damage [3]. Endo et al. [23] showed that PPIs were the risk factors for LDA-induced small intestinal damage. In addition, a meta-analysis performed by Lo and Chan [24] indicated that the use of PPIs was statistically associated with SIBO. Thus, PPIs are closely associated with the pathogenesis of dysbiosis and may exacerbate the NSAID-induced small intestinal damage. Further prospective studies are required to investigate the association between PPIs and NSAID-induced small intestinal damages.

\section{Probiotics Prevents NSAID-Induced Small Intestinal Damage}

Some antibiotics are effective in preventing the NSAID-induced small intestinal damage. However, NSAIDs are frequently prescribed for a long period to control chronic pain, and the long-term use of antibiotics increases the risk of development of multi-drug resistant bacteria and induction of microbial substitution-associated enteritis. Therefore, agents modulating the microbiota could be better candidates for treating NSAID-induced enteropathy.

The preventive effect of probiotics has been reported in GI tract disorders such as inflammatory bowel disease [25] and irritable bowel syndrome [26]. Lactobacillus casei strain Shirota (LcS) is a probiotic bacterium originally isolated from humans [27]. We examined the preventive effect of $\mathrm{LCS}$ on indomethacin-induced small intestinal damage in rats [28]. Our results showed that treatment with viable LcS for one week ameliorated the indomethacin-induced small intestinal damage and suppressed neutrophil infiltration and expression of inflammatory cytokines, including TNF- $\alpha$. In addition, L-lactic acid produced by LcS suppressed indomethacin-induced small intestinal damage. Both of LcS and L-lactic acid culture supernatant inhibited NF- $\kappa B$ activation and increased TNF- $\alpha$ expression in LPS-treated THP-1 cells, the human monocytic cell line. These findings suggest that LcS has a prophylactic effect on indomethacin-induced enteropathy and this probiotic effect of LcS is mediated by L-lactic acid.

Montalto et al. [29] reported that the results of a clinical trial in which treatment with a probiotic mixture significantly decreased the fecal calprotectin concentration in healthy volunteers receiving indomethacin compared to those receiving placebo. Endo et al. [30] showed that treatment with $L$. casei significantly decreased the number of intestinal mucosal breaks assessed by video capsule endoscopy in patients taking LDA compared to those receiving control treatment.

\section{Rebamipide Inhibits NSAID-Induced Small Intestinal Damage by Modulating the Composition of the Intestinal Microbiota}

Rebamipide ((2RS)-2-(4-chlorobenzoylamino)-3-(2oxo-1,2-dihydroquinolin-4-yl) propanoic acid) is a mucoprotective drug that has been clinically used for treating gastritis and peptic ulcers [31,32]. Rebamipide has preventive effects on NSAID-induced small intestinal damage in addition to upper GI tract damages [33-35]. A previous randomized placebo-controlled study showed that rebamipide was effective in preventing LDA-induced small intestinal damage [36]. Although rebamipide increases $\mathrm{PG}$ production and scavenges free radicals, wheth- 
er rebamipide modulates the composition of the intestinal microbiota has not been elucidated thus far.

We investigated the effects of rebamipide on experimental indomethacin-induced small intestinal damage and the composition of small intestinal microbiota in mice [37]. We found that damaged lesions in the small intestine were significantly smaller in rebamipide-treated mice than in vehicle-treated mice. The contents of small intestine were subjected to terminal restriction fragment length polymorphism (T-RFLP) analysis to assess the diversity of enterobacteria, and the results showed that rebamipide increased the percentage of Lactobacillales and decreased the percentage of Bacteroides and Clostridium subcluster XIVa. Compared to vehicle-treated, rebamipide-treated mice showed an increase in the expression of $\alpha$-defensin (cryptdin) 5, an important anti-microbial peptide produced by Paneth cells for the modulation of small intestinal microbiota [38]. These findings suggest that rebamipide inhibits indomethacin-induced small intestinal damage possibly by modulating the composition of the intestinal microbiota through the upregulation of $\alpha$-defensin 5 .

Imaeda et al. [39] reported that the combination of indomethacin and rebamipide significantly altered the composition of the gut microbiota in mice characterized by an increase in Bifidobacteriales and Prevotella using T-RFLP analysis. They concluded that the activity of rebamipide on NSAID-induced small intestinal damage might be through the modulation of the composition of the gut microbiota.

T-RFLP analysis has limitations in that it is a semiquantitative analysis, which detects the proportion of dominant bacterial species by cluster structure. Thus, metagenome analysis using a next-generation sequencer will provide further information about the rebamipide-induced modulation of the composition of the microbiota.

\section{Conclusion}

The strategy for prevention and treatment for NSAIDinduced small intestinal damage has not been established thus far, and further studies are required to elucidate the mechanisms underlying this damage. Accumulating evidence suggest that the microbiota plays a key role in NSAID-induced small intestinal damage, and modulation of the composition of the intestinal microbiota could be a new therapeutic strategy for such damage. Metagenome analysis of the small intestinal microbiota using next-generation sequencer and basic research using human-flora-associated mice will enable further understanding of the role of small intestinal microbiota in NSAID-induced small intestinal damage, and prospective randomized clinical trials will provide insights into the treatment and prophylaxis of this condition.

\section{Disclosure Statement}

The authors declare no conflicts of interest for this review article.

\section{References}

1 Graham DY, Opekun AR, Willingham FF, Qureshi WA: Visible small-intestinal mucosal injury in chronic NSAID users. Clin Gastroenterol Hepatol 2005;3:55-59.

-2 Sugimori S, Watanabe T, Tabuchi M, Kameda $\mathrm{N}$, Machida $\mathrm{H}$, Okazaki $\mathrm{H}$, Tanigawa $\mathrm{T}$, Yamagami H, Shiba M, Watanabe K, Tominaga K, Fujiwara Y, Oshitani N, Koike T, Higuchi $\mathrm{K}$, Arakawa T: Evaluation of small bowel injury in patients with rheumatoid arthritis by capsule endoscopy: effects of anti-rheumatoid arthritis drugs. Digestion 2008;78:208-213.

-3 Watanabe T, Tanigawa T, Nadatani Y, Nagami Y, Sugimori S, Okazaki H, Yamagami H, Watanabe K, Tominaga K, Fujiwara Y, Koike T, Arakawa T: Risk factors for severe nonsteroidal anti-inflammatory drug-induced small intestinal damage. Dig Liver Dis 2013;45:390-395.

-4 Gu S, Chen D, Zhang JN, Lv X, Wang K, Duan LP, Nie Y, Wu XL: Bacterial community map- ping of the mouse gastrointestinal tract. PLoS One 2013;8:e74957.

5 Zoetendal EG, Raes J, van den Bogert B, Arumugam M, Booijink CC, Troost FJ, Bork P, Wels M, de Vos WM, Kleerebezem M: The human small intestinal microbiota is driven by rapid uptake and conversion of simple carbohydrates. ISME J 2012;6:14151426.

-6 Robert A, Asano T: Resistance of germfree rats to indomethacin-induced intestinal lesions. Prostaglandins 1977;14:333-341.

7 Uejima M, Kinouchi T, Kataoka K, Hiraoka I, Ohnishi Y: Role of intestinal bacteria in ileal ulcer formation in rats treated with a nonsteroidal antiinflammatory drug. Microbiol Immunol 1996;40:553-560.

8 Konaka A, Kato S, Tanaka A, Kunikata T, Korolkiewicz R, Takeuchi K: Roles of enterobacteria, nitric oxide and neutrophil in pathogen- esis of indomethacin-induced small intestinal lesions in rats. Pharmacol Res 1999;40:517524.

99 Takeuchi K, Satoh H: NSAID-induced small intestinal damage - roles of various pathogenic factors. Digestion 2015;91:218-232.

10 Watanabe T, Higuchi K, Kobata A, Nishio H, Tanigawa T, Shiba M, Tominaga K, Fujiwara Y, Oshitani N, Asahara T, Nomoto K, Takeuchi K, Arakawa T: Non-steroidal anti-inflammatory drug-induced small intestinal damage is toll-like receptor 4 dependent. Gut 2008;57: 181-187.

11 Nadatani Y, Watanabe T, Tanigawa T, Machida $H$, Okazaki $H$, Yamagami $H$, Watanabe K, Tominaga K, Fujiwara Y, Arakawa T: High mobility group box 1 promotes small intestinal damage induced by nonsteroidal anti-inflammatory drugs through toll-like receptor 4. Am J Pathol 2012;181:98-110. 
$\checkmark 12$ Higashimori A, Watanabe T, Nadatani Y, Takeda S, Otani K, Tanigawa T, Yamagami H, Shiba M, Tominaga K, Fujiwara Y, Arakawa T: Mechanisms of NLRP3 inflammasome activation and its role in NSAID-induced enteropathy. Mucosal Immunol 2016;9:659-668.

13 Watanabe T, Tanigawa T, Nadatani Y, Otani $\mathrm{K}$, Machida $\mathrm{H}$, Okazaki $\mathrm{H}$, Yamagami $\mathrm{H}$, Watanabe K, Tominaga K, Fujiwara Y, Arakawa T: Mitochondrial disorders in NSAIDsinduced small bowel injury. J Clin Biochem Nutr 2011;48:117-121.

14 Bai K: On the mechanism of cereobiogen readjustment to dysbiosis. Prog Clin Biol Res 1985;181:169-170.

$\checkmark 15$ Frank DN, Robertson CE, Hamm CM, Kpadeh Z, Zhang T, Chen H, Zhu W, Sartor RB, Boedeker EC, Harpaz N, Pace NR, Li E: Disease phenotype and genotype are associated with shifts in intestinal-associated microbiota in inflammatory bowel diseases. Inflamm Bowel Dis 2011;17:179-184.

16 Tamboli CP, Neut C, Desreumaux P, Colombel JF: Dysbiosis in inflammatory bowel disease. Gut 2004;53:1-4.

17 Cani PD, Bibiloni R, Knauf C, Waget A, Neyrinck AM, Delzenne NM, Burcelin R: Changes in gut microbiota control metabolic endotoxemia-induced inflammation in high-fat diet-induced obesity and diabetes in mice. Diabetes 2008;57:1470-1481.

18 Ley RE, Turnbaugh PJ, Klein S, Gordon JI: Microbial ecology: human gut microbes associated with obesity. Nature 2006;444:10221023.

19 Henao-Mejia J, Elinav E, Jin C, Hao L, Mehal WZ, Strowig T, Thaiss CA, Kau AL, Eisenbarth SC, Jurczak MJ, Camporez JP, Shulman GI, Gordon JI, Hoffman HM, Flavell RA: Inflammasome-mediated dysbiosis regulates progression of NAFLD and obesity. Nature 2012;482:179-185.

20 Donaldson RM Jr: Normal bacterial populations of the intestine and their relation to intestinal function. N Engl J Med 1964;270 1050-1056.

-21 Muraki M, Fujiwara Y, Machida H, Okazaki $\mathrm{H}$, Sogawa M, Yamagami H, Tanigawa T, Shiba M, Watanabe K, Tominaga K, Watanabe T, Arakawa T: Role of small intestinal bacterial overgrowth in severe small intestinal damage in chronic non-steroidal anti-inflammatory drug users. Scand J Gastroenterol 2014;49: 267-273.

22 Wallace JL, Syer S, Denou E, de Palma G, Vong L, McKnight W, Jury J, Bolla M, Bercik P, Collins SM, Verdu E, Ongini E: Proton pump inhibitors exacerbate NSAID-induced small intestinal injury by inducing dysbiosis. Gastroenterology 2011; 141: 1314-1322, e1311-e1315.

23 Endo H, Sakai E, Taniguchi L, Kessoku T, Komiya Y, Ezuka A, Kawamura H, Taguri M, Higurashi T, Ohkubo H, Yamada E, Takahashi H, Inamori M, Maeda S, Sakaguchi T, Hata Y, Nagase H, Nakajima A: Risk factors for small-bowel mucosal breaks in chronic low-dose aspirin users: data from a prospective multicenter capsule endoscopy registry. Gastrointest Endosc 2014;80:826834.

24 Lo WK, Chan WW: Proton pump inhibitor use and the risk of small intestinal bacterial overgrowth: a meta-analysis. Clin Gastroenterol Hepatol 2013;11:483-490.

25 Bibiloni R, Fedorak RN, Tannock GW, Madsen KL, Gionchetti P, Campieri M, De Simone C, Sartor RB: VSL\#3 probiotic-mixture induces remission in patients with active ulcerative colitis. Am J Gastroenterol 2005;100: 1539-1546.

26 O’Mahony L, McCarthy J, Kelly P, Hurley G, Luo F, Chen K, O'Sullivan GC, Kiely B, Collins JK, Shanahan F, Quigley EM: Lactobacillus and bifidobacterium in irritable bowel syndrome: symptom responses and relationship to cytokine profiles. Gastroenterology 2005; 128:541-551.

27 Matsuzaki T: Immunomodulation by treatment with lactobacillus casei strain shirota Int J Food Microbiol 1998;41:133-140.

28 Watanabe T, Nishio H, Tanigawa T, Yamagami $\mathrm{H}$, Okazaki $\mathrm{H}$, Watanabe $\mathrm{K}$, Tominaga K, Fujiwara Y, Oshitani N, Asahara T, Nomoto K, Higuchi K, Takeuchi K, Arakawa T: Probiotic lactobacillus casei strain shirota prevents indomethacin-induced small intestinal injury: involvement of lactic acid. Am J Physiol Gastrointest Liver Physiol 2009;297:G506G513.

29 Montalto M, Gallo A, Curigliano V, D’Onofrio F, Santoro L, Covino M, Dalvai S, Gasbarrini A, Gasbarrini G: Clinical trial: the effects of a probiotic mixture on non-steroidal anti-inflammatory drug enteropathy - a randomized, double-blind, cross-over, placebo-controlled study. Aliment Pharmacol Ther 2010; 32:209-214.

30 Endo H, Higurashi T, Hosono K, Sakai E, Sekino Y, Iida H, Sakamoto Y, Koide T, Takahashi $\mathrm{H}$, Yoneda $\mathrm{M}$, Tokoro $\mathrm{C}$, Inamori $\mathrm{M}$, Abe Y, Nakajima A: Efficacy of lactobacillus casei treatment on small bowel injury in chronic low-dose aspirin users: a pilot randomized controlled study. J Gastroenterol 2011;46:894-905.
31 Arakawa T, Kobayashi K, Yoshikawa T, Tarnawski A: Rebamipide: overview of its mechanisms of action and efficacy in mucosal protection and ulcer healing. Dig Dis Sci 1998; 43(9 suppl):5S-13S.

-32 Arakawa T, Higuchi K, Fujiwara Y, Watanabe T, Tominaga K, Sasaki E, Oshitani N, Yoshikawa T, Tarnawski AS: 15th anniversary of rebamipide: looking ahead to the new mechanisms and new applications. Dig Dis Sci 2005; 50(suppl 1):S3-S11.

-33 Mizoguchi H, Ogawa Y, Kanatsu K, Tanaka A, Kato S, Takeuchi K: Protective effect of rebamipide on indomethacin-induced intestinal damage in rats. J Gastroenterol Hepatol 2001;16:1112-1119.

34 Niwa Y, Nakamura M, Ohmiya N, Maeda O, Ando T, Itoh A, Hirooka Y, Goto H: Efficacy of rebamipide for diclofenac-induced smallintestinal mucosal injuries in healthy subjects: a prospective, randomized, double-blinded, placebo-controlled, cross-over study. J Gastroenterol 2008;43:270-276.

35 Fujimori S, Takahashi Y, Gudis K, Seo T, Ehara A, Kobayashi T, Mitsui K, Yonezawa $M$, Tanaka S, Tatsuguchi A, Sakamoto C: Rebamipide has the potential to reduce the intensity of NSAID-induced small intestinal injury: a double-blind, randomized, controlled trial evaluated by capsule endoscopy. J Gastroenterol 2011;46:57-64.

-36 Watanabe T, Takeuchi T, Handa O, Sakata Y, Tanigawa T, Shiba M, Naito Y, Higuchi K, Fujimoto K, Yoshikawa T, Arakawa T: A multicenter, randomized, double-blind, placebocontrolled trial of high-dose rebamipide treatment for low-dose aspirin-induced moderate-to-severe small intestinal damage. PLoS One 2015; 10:e0122330.

-37 Tanigawa T, Watanabe T, Otani K, Nadatani Y, Ohkawa F, Sogawa M, Yamagami H, Shiba M, Watanabe K, Tominaga K, Fujiwara Y, Takeuchi K, Arakawa T: Rebamipide inhibits indomethacin-induced small intestinal injury: possible involvement of intestinal microbiota modulation by upregulation of a-defensin 5. Eur J Pharmacol 2013;704:6469.

38 Ouellette AJ: Defensin-mediated innate immunity in the small intestine. Best Pract Res Clin Gastroenterol 2004;18:405-419.

39 Imaeda H, Fujimoto T, Takahashi K, Kasumi E, Fujiyama Y, Andoh A: Terminal-restriction fragment length polymorphism (T-RFLP) analysis for changes in the gut $\mathrm{mi}-$ crobiota profiles of indomethacin- and rebamipide-treated mice. Digestion 2012;86: 250-257. 